\title{
Simulation of Racing Greyhound Kinematics
}

\author{
Md Imam Hossain $\mathbb{1}^{\mathrm{a}}$, David Eager $\mathbb{1}^{\mathrm{b}}$ and Paul Walker $\mathbb{C}^{\mathrm{c}}$ \\ Faculty of Engineering and Information Technology, University of Technology Sydney, Sydney, \\ PO Box 123, Broadway 2007, Australia \\ MDImam.Hossain@uts.edu.au,David.Eager@uts.edu.au,Paul.Walker@uts.edu.au
}

\begin{abstract}
Keywords: Greyhound Racing, Greyhound Kinematics, Dynamic Simulation, Numerical Simulation, Rigid Body Dynamics, Injury Prevention, Animal Welfare.

Abstract: $\quad$ This paper outlines greyhound dynamics results for yaw rate, speed, and the congestion pattern during a race derived through numerical modelling. The simulation results presented are also correlated to actual race data to validate modelling performance and reliability. The tasks carried out include the development of a numerical model for greyhound veering and race related supporting models, creating track 3D models replicated from actual survey data of the track, establishing a simulation environment that emulates an actual greyhound race, and the processing of both simulation and actual race data. The results show that greyhounds are susceptible to experience varying high acceleration in first five seconds into the race, during which a minimum average forward acceleration of $3.9 \mathrm{~m} / \mathrm{s}^{2}$ was calculated, a peak yaw rate magnitude of $0.4 \mathrm{rad} / \mathrm{s}$ before the bend while transitioning into the track, and congestion during a race is affected by lure driving performance.
\end{abstract}

\section{INTRODUCTION}

Greyhound racing is a popular sport in many countries where the industry is thriving. Gradually, focus is changing to best-racing performance outcomes while minimising race injuries on the tracks. As a result, simulation of greyhound racing becoming an increasingly important technique for evaluating races as it would directly and indirectly benefit many parties including track designers, race operations managers to name few.

When it comes to the greyhound, they are able to travel by a maximum speed averaging above $70 \mathrm{~km} / \mathrm{h}$ on the land. This astonishing speed is achieved through galloping gait of a greyhound which is also preferred gait for most quadrupedal mammals (Krasny, and Orin, 2004). However, musculoskeletal injuries are a common happening in racing quadrupeds such as greyhounds when compared to non-racing quadrupeds (Sicard et al., 1999). A study showed that various track dynamic conditions, as well as greyhound running speed, have a significant influence on race performance and injuries (Iddon et al., 2014, Mahadavi et al., 2018, Hasti et al., 2017a). Likewise, investigation of five tracks showed that factors such as track surface grades, achievable speed, and race distance resulted significant effect on greyhound injury rates (Iddon et al., 2014). Moreover, various observations of racing greyhound injuries indicated that congestion at the start of the race as well as at the immediate bend in the track is a precursor to major race injuries (Hayati et al., 2017b).

For greyhound racing, a traditional approach for analysing race dynamics can be cumbersome and difficult to achieve. This is because traditional techniques such as graphical and analytical methods sometimes lack accuracy and the complexity can increase exponentially for a relatively simple practical problem (Garcia et al., 2012). An alternative solution is numerical analysis and methods through fast processing of alphanumeric data, data formulation and computational methods (Garcia et al., 2012). In the numerical approach, mathematical models are developed from observation of physical and technical processes where derived equations are computed at discrete points in the time (Griebel et al., 2010). Furthermore, the availability of corresponding

(D) https://orcid.org/0000-0002-1246-3454

b (D) https://orcid.org/0000-0003-1926-7867

c(D) https://orcid.org/0000-0003-3988-3966 
physical or technical processes results would allow verification of numerical results or improvements to developed numerical model methods (Griebel et al., 2010).

This paper describes the design and mechanism of dynamic numerical models for conducting greyhound racing simulation which is processing efficient and yet robust enough to extract various greyhound kinematics and racing dynamics, which include greyhound yaw rate, speed, congestion pattern, greyhound path following as well as factors which affect racing performance. Furthermore, the design and mechanism described can be expanded and utilised in other prospective areas such as vehicle and quadruped running simulations with multiple interacting factors having proper constraints in place. The realistic constraints are such that any dynamic system models developed exhibit controllability of the numerical algorithms output convergence (Respondek, 2010). This is because controllability of dynamical systems allows extension of dynamic systems conditions for given problems (Respondek, 2005).

Moreover, this paper analyses simulation and actual race data for deriving trends in racing greyhounds. The simulation carried out were matched to available field data configuration such as eight greyhounds running over a defined distance in a track which has two bends track paths as well as two straights track paths.

\section{MATHEMATICAL MODEL DEVELOPMENT OVERVIEW}

To create a viable model for greyhound racing, there are various variables which first need to be identified and addressed. These variables may come from within the racing track or from the greyhound. By considering racing track and greyhound as different systems their inherent system behaviour defining variables can be identified. Furthermore, race operational configuration and running conditions define a system which by emulating it creates necessary inputs for a simulation model of greyhound racing.

\subsection{Model Scope}

When a greyhound is racing, its motion can be defined and traced in term of displacement, velocity, and acceleration in Euclidean space. While the nominal acceleration of greyhound can be directly related to forces acting on it, deriving of the instantaneous displacement and velocity of greyhound can be a complex task. This is because the instantaneous displacement and velocity of a greyhound in racing not only depend on the racing track design and racing operational running conditions but also rely on the adjacent greyhounds' dynamic profiles. This creates a greater unpredictability in the controllability of a greyhound's instantaneous displacement and velocity during a race. Furthermore, observation has confirmed that a racing greyhound can bump into another adjacent greyhound as well as follow a particular path, which is not defined by its motion limiting force factors but are an inherent part of race dynamics. Consequently, the interactions between greyhounds in a race have a significant impact on the greyhound race dynamics. A greyhound veering model is developed which outputs greyhound locations during a race from the start of the race till the end. The model predicts the path taken by individual greyhounds during a race while having each greyhound its own character in terms of velocities. Finally, the model calculates adjacent greyhounds' locational, track design and race operational influences and optimises potential locations of greyhound during a race. In the simplest form, the model can be described by a finite dimensional system state equation. This model is said to be controllable if and only if the control variable would allow the system to reach any final state in the control space in the finite time from any given initial state (Respondek, 2005).

\subsection{Understanding of Greyhound Racing Elements}

There are a number of distinct elements which take part in a greyhound racing. While some of these racing elements are dynamic in nature, there are also elements which are static and have a great impact on a racing greyhound. The main dynamic bodies in racing are the lure during a race, adjacent racing greyhounds, and individual greyhound limbs which are required for greyhound locomotion. The fundamental static bodies relating to racing are race starting boxes locations and orientations relative to track, track surface properties including surface traction, impact attenuation qualities, track camber, and track racing line. During a race, lure, starting boxes, and track are regulated to certain degrees that their behaviours are controlled and follow a specific predefined pattern. 


\subsection{Model Composition and Mechanism}

By considering a greyhound as a point object its overall motion in a race can be formulated by knowing the factors which induce the motion as well as the factors which alter the motion characteristics as a result of combined racing environmental and greyhound limits. The main driving factor for greyhound motion is greyhound stride while the motion altering factors are greyhound natural steering limits, collision in a race, track geometry and surface condition, lure velocity profile and track boundaries. These discrete factors are modelled using kinematics and vectors equations where kinetics equations and input to output unit tests are used for finding initial and final values for the model equations. Superposition principle is applied to the model equations to solve for aggregated results of discrete race dynamics factors and calculate greyhound location during a race using Euler's method. The summation of discreet factors can be expressed as

$$
\begin{gathered}
\boldsymbol{V}\left(\mathrm{v}_{1}+\mathrm{v}_{2}+\mathrm{v}_{3}+\mathrm{v}_{4}+\mathrm{v}_{5}+\mathrm{v}_{6}+\mathrm{v}_{7}+\mathrm{v}_{8}+\mathrm{v}_{9}\right) \\
=\boldsymbol{V}\left(\mathrm{v}_{1}\right)+\boldsymbol{V}\left(\mathrm{v}_{2}\right)+\boldsymbol{V}\left(\mathrm{v}_{3}\right)+\boldsymbol{V}\left(\mathrm{v}_{4}\right)+\boldsymbol{V}\left(\mathrm{v}_{5}\right)+ \\
\boldsymbol{V}\left(\mathrm{v}_{6}\right)+\boldsymbol{V}\left(\mathrm{v}_{7}\right)+\boldsymbol{V}\left(\mathrm{v}_{8}\right)+\boldsymbol{V}\left(\mathrm{v}_{9}\right)
\end{gathered}
$$

In which $\boldsymbol{V}$ is the final velocity vector function for $v_{1} \ldots v_{9}$ discrete factors of race dynamics. The $v_{1}$ factor represents greyhound stride acceleration velocity due to the sum of all the forces exerted by a greyhound's stride which can be modelled using Newton's second law (2). This is also the forward acceleration of greyhound. It was assumed that a greyhound's exerted stride force remains constant for the entire race duration although, in reality, data show greyhound acceleration is highly variable during the first three strides. However, as the time fraction of the first three strides is relatively small compared to entire race duration, a linear acceleration model and an averaged value of greyhound maximum accelerations are appropriate for getting stable final velocity output. For constant acceleration, greyhound maximum forward acceleration is calculated using Eq. 3 where greyhound final velocity (v) is $19.4 \mathrm{~m} / \mathrm{s}$, greyhound displacement (s) for reaching final velocity is $15 \mathrm{~m}$ and initial velocity $\left(\mathrm{v}_{0}\right)$ is $0 \mathrm{~m} / \mathrm{s}$. This maximum forward acceleration is plugged into Eq. 4 to calculate the greyhound stride acceleration velocity factor $v_{1}$. In Eq. 4, $v_{0}$ is greyhound velocity in previous time instant and $\mathrm{dt}$ is the time elapsed between $\mathrm{v}$ and $\mathrm{v}_{0}$.

$$
\Sigma F=m_{\mathrm{s}}
$$

$$
\begin{gathered}
\mathrm{v} 2=\mathrm{v}_{0}^{2}+2 * \mathrm{a}_{\mathrm{s}} * \mathrm{~s} \\
\mathrm{v}=\mathrm{v}_{0}+\mathrm{a}_{\mathrm{s}} * \mathrm{dt}
\end{gathered}
$$

The purpose of the $\mathrm{v}_{2}$ factor is to provide greyhound the motivation to race as well as steer greyhound heading as it moves in the track. Therefore, this factor is a function of greyhound's lure line of sight, frictional force, and centrifugal force vectors. However, observation of greyhound race and race data have confirmed that lure line of sight is a significant guiding influence for greyhound path following around the track in absence of other influences such as congestions due to adjacent racing greyhounds. As a result, the lure line of sight vector was considered also a function of frictional force, and centrifugal force vectors (Eq. 5). The lure line of sight vector is constructed from lure and greyhound instantaneous locations in the track. The frictional force has two components where one is due to track camber $(\theta)$ and another one is the nominal friction due to greyhound instantaneous velocity as denoted by $\mathrm{m}$ $* \mathrm{~g} * \sin \theta$ and $\mathrm{C} * \mathrm{v}$ respectively.

Lure line of sight vector $=$ lure line of sight vector + centrifugal force vector + paw and track surface frictional force vector Centrifugal force $=\mathrm{m} * \mathrm{v}^{2} / \mathrm{R}$

Greyhound paw and track surface frictional force $=(\mathrm{m} * \mathrm{~g} * \sin \theta)+(\mathrm{C} * \mathrm{v})$

In Eq. 6, $\mathrm{R}$ is instantaneous radius of curvature of greyhound path following and $\mathrm{m}$ is greyhound mass. In Eq. 7, $\mathrm{m}$ is greyhound mass and $\mathrm{C}$ is a constant.

The $v_{3}$ factor is a tweaking vector to $v_{2}$ factor to achieve precise greyhound heading movement without which results in unnatural greyhound heading movement behaviour. The reason for this, in realworld greyhound heading direction change does not go through step change to meet the lure following path instead it goes through many mini-movements to catch up lure following path. This creates a lag between greyhound spotting lure location and greyhound merging with the lure following path. Moreover, greyhound has physical turning limits at an instant. All of these phenomena are modelled using a steering vector which is a function of greyhound current heading direction vector and lure line of sight vector.

Steering vector $=$ lure line of sight vector current heading direction vector 
The $v_{4}$ final velocity factor determines the outcome of greyhound checking and bumping as well as greyhound collision avoidance tendency to adjacent greyhound during a race which results in greyhound surrounding aware variable velocities. These situations in a race are modelled by using a collision avoidance vector which successively finds greyhounds in proximity and through a number of iterations construct a clearance vector. The exact number of iterations was chosen based on simulation time stamps and convergence of the clearance vector. Furthermore, by assuming there is no collision between two greyhounds vertically, circle to circle collision detection is used for checking overlapping greyhounds.

Collision avoidance vector $=$ location vector of greyhound in proximity - location vector of greyhound

Clearance vector $=$ current heading direction vector - collision avoidance vector

The $v_{5}$ factor finds the effect of track cross falls on greyhound veering. As the effect of cross fall can be proportional to track banking angle $(\theta)$ at spot location, a linear calibrated force is used along with normal force vector for calculation. To find banking angle at a given location in track, the track surface is discretised using small triangles where the vertices of each triangle are used for finding normal force vector $(\mathrm{N})$ and corresponding bangle angle $(\theta)$.

$$
\begin{aligned}
& \mathrm{N}_{\mathrm{x}}=\mathrm{U}_{\mathrm{y}} * \mathrm{~V}_{\mathrm{z}}-\mathrm{U}_{\mathrm{z}} * \mathrm{~V}_{\mathrm{y}} \\
& \mathrm{N}_{\mathrm{y}}=\mathrm{U}_{\mathrm{z}} * \mathrm{~V}_{\mathrm{x}}-\mathrm{U}_{\mathrm{x}} * \mathrm{~V}_{\mathrm{z}} \\
& \mathrm{N}_{\mathrm{z}}=\mathrm{U}_{\mathrm{x}} * \mathrm{~V}_{\mathrm{y}}-\mathrm{U}_{\mathrm{y}} * \mathrm{~V}_{\mathrm{x}}
\end{aligned}
$$

Normal force vector $(\mathrm{N})$ magnitude $=$

$$
\mathrm{m} * \mathrm{~g} * \cos \theta+(\mathrm{C} * \mathrm{v})
$$

Where, $U=p_{2}-p_{1}$ and $V=p_{3}-p_{1}$ for $p_{1}, p_{2}, p_{3}$ triangle vertices and $\mathrm{m}$ is greyhound mass and $\mathrm{C}$ is a constant.

While racing greyhound avoids colliding with track boundaries like inside lure rail. The $\mathrm{v}_{6}$ factor is used for applying a track boundary collision avoidance vector to final velocity vector. For this purpose, track boundaries are sampled with a number of evenly spaced points and by using nearest points to greyhound location boundary collision avoidance vector is found.

Boundary collision avoidance vector $=$ location vectors of adjacent points on track boundaries - location vector of greyhound
The $\mathrm{V}_{7}$ factor models variable track surface conditions at different track locations as well as variable greyhound stride acceleration from greyhound to greyhound for the race period. Modelling of variable track surface conditions is essential, as, despite identical stride from a greyhound over the race period, non-uniform track surface conditions such as hardness, softness, and coefficient of friction determine greyhound stride acceleration. This factor is a function of stride duration, race time, and a random number generator.

The $\mathrm{v}_{8}$ factor adds a specific greyhound behaviour to final velocity vector which occurs when a greyhound is lagging behind the lure significantly as observed from various races. It was noticeable that greyhound maintains an additional offset distance from track inside boundary to get a better sight of the lure depending on the distance between greyhound and lure. However, various races also indicate that this specific behaviour varies from greyhound to greyhound. This situation is modelled by constructing a boundary offset vector which is a function of greyhound distance to lure, minimum offset from the boundary and a constant as given below:

$$
\begin{aligned}
& \text { Boundary offset vector }=\text { minimum offset } \\
& \text { from boundary } *(\text { distance to lure } / \mathrm{C})
\end{aligned}
$$

Where minimum offset from boundary and $\mathrm{C}$ are calibrated to be $0.5 \mathrm{~m}$ and $5 \mathrm{~m}$ respectively.

The $v_{9}$ factor defines lure motion in terms of track path and leading greyhound location in the race. For this factor, a model function is created which takes into account of track predefined lure travel path and lure and leading greyhound separation distance to provide lure instantaneous velocity which would maintain lure driving for the duration of a race while maintaining a separation distance. The model function first calculates heading direction of the lure by copying track curvature profile and then set lure instantaneous speed based on the lure and leading greyhound separation distance. The setting of lure instantaneous speed (S) is based on the following rules where the constants were tweaked to meet the lure driving performance:

$$
\begin{gathered}
((\mathrm{A}>\mathrm{B} \rightarrow(\mathrm{C}>30 \rightarrow \mathrm{X}=1.9489)) \wedge(\mathrm{A}>\mathrm{B} \\
\rightarrow(\mathrm{C}>20 \rightarrow \mathrm{X}=3.3378)) \wedge(\mathrm{A}>\mathrm{B} \rightarrow(\mathrm{C}> \\
15 \rightarrow \mathrm{X}=6.1156)) \wedge(\mathrm{A}>\mathrm{B} \rightarrow(\mathrm{C}>10 \rightarrow \mathrm{X} \\
=8.893)) \wedge(\mathrm{A}>\mathrm{B} \rightarrow(\mathrm{C}>5 \rightarrow \mathrm{X}= \\
16.9489)) \wedge(\mathrm{A}>\mathrm{B} \rightarrow(\mathrm{C}>1 \rightarrow \mathrm{X}=17.504)) \\
\wedge(\mathrm{A}>\mathrm{B} \rightarrow(\mathrm{C}>0.2 \rightarrow \mathrm{X}=17.782))) \wedge((\mathrm{A} \\
<\mathrm{B} \rightarrow(\mathrm{C}>14 \rightarrow \mathrm{X}=14)) \wedge(\mathrm{A}<\mathrm{B} \rightarrow(\mathrm{C}> \\
10 \rightarrow \mathrm{X}=18.62) \wedge(\mathrm{A}<\mathrm{B} \rightarrow(\mathrm{C}>5 \rightarrow \mathrm{X}= \\
17.504)) \wedge(\mathrm{A}<\mathrm{B} \rightarrow(\mathrm{C}>1 \rightarrow \mathrm{X}=16.9489)) \\
\wedge(\mathrm{A}<\mathrm{B} \rightarrow(\mathrm{C}>0.2 \rightarrow \mathrm{X}=20))
\end{gathered}
$$


In which $\mathrm{A}$ is distance between leading greyhound and lure, $\mathrm{B}$ is maintaining separation distance, $\mathrm{C}$ is the difference between $\mathrm{A}$ and $\mathrm{B}$, and finally $\mathrm{X}$ is lure instantaneous speed.

Finally, in the greyhound motion model composition, vertical acceleration velocity factor was neglected since instantaneous vertical velocity is negligible compared to other greyhound velocity influencing factors because of low track surface penetration by greyhound paw during galloping in a race.

\section{SIMULATION PLATFORM}

The Blender software package was used as the simulation platform for creating contents for simulation visualisation as well as implementation, setup and data extraction of the simulation models using Python programming language. First, race track 3D model was imported into Blender virtual Euclidean space from track survey data where it is constructed and formatted to meet the needs of mathematical models. Then, racing elements 3D models such as starting boxes, greyhounds, lure and shape defining objects such as track boundary curves were created in Blender virtual Euclidean space meeting mathematical model requirements. Finally, the Blender Python application programming interface was used for writing simulation code inside Blender software package. The primary components of simulation code are: greyhound object which defines a greyhound's dynamic model as well as it's state in a particular time stamp, a lure object which defines lure dynamic model and its state in a particular time stamp, a method for calculating collision between greyhounds, and a method containing code for simulation numerical solver and updating 3D models in Blender virtual Euclidean space. For both lure and greyhounds' motions, the numerical solver calculates time-varying behaviour of each dynamic system by solving models functions and numerical integration using Euler method where the results from each model function are compounded using superposition principle. For example, the final location of each dynamic object is determined by integrating the velocity over time as follows:

$$
\begin{gathered}
\mathrm{S}=\int \mathrm{v} * \mathrm{dt} \\
\mathrm{S}_{\text {new }}=\mathrm{S}_{\text {old }}+\mathrm{v} * \mathrm{dt}
\end{gathered}
$$

Where $S_{\text {new }}$ is the new location, $S_{\text {old }}$ is the old location, $\mathrm{v}$ is instantaneous velocity and $\mathrm{dt}$ is the smallest unit of time in simulation.
The global variables in the simulation setup are lure initial speed, lure maintaining acceleration, lure starting acceleration, greyhound maximum average acceleration, greyhound maximum speed, greyhound minimum speed, lure greyhound separation distance, starting boxes locations and orientations, and lure offset from the starting boxes before racing. These variables were adjusted to match greyhound races.

\section{SIMULATION RESULTS AND PERFORMANCE EVALUATION}

To validate greyhound racing model simulation results greyhound race data were retrieved from IsoLynx system. The IsoLynx system captures realtime greyhound coordinates data during a race where it can trace a greyhound's location in the track in terms of $\mathrm{X}$ and $\mathrm{Y}$ coordinates relative signal towers. Different races greyhound coordinate data from both simulation results and actual race were used for validating simulation models performances. The races which were selected from both simulation and actual race have identical setups in terms racing time of the day, racing distance to rule out unknown factors affecting the comparisons as well as to find different racing factors general trends. Moreover, simulation and race data were resampled to match greyhound stride duration since greyhound dynamics state is reflected with every stride. Finally, simulations were run with slightly varying lure driving, greyhound maximum acceleration behaviour configurations from nominal values to exaggerate the effects of different racing factors outcomes. The following sections analyses model simulation performance to race data.

\subsection{Greyhound Performance Indicators}

The following major greyhound kinematics variables were analysed.

\subsubsection{Path Following}

As greyhound races, they follow a specific path in the track. The following graphs show $\mathrm{X}$ and $\mathrm{Y}$ coordinates of a single greyhound during a race. The graphs show that greyhound path coordinates shape from simulation model results closely match with race data. By looking into rate change of coordinates subtle differences were also analysed. The maximum and minimum percentage differences between simulation and race for $\mathrm{X}$ coordinates are $6 \%$ and $7 \%$ respectively. The maximum and minimum 
percentage differences between simulation and race for $\mathrm{Y}$ coordinates are $4 \%$ and $15 \%$ respectively. This shows simulated models resulted in a highly accurate path following while percentages differences can be attributed to slightly different race configurations between simulation and actual race and varying nature of each race.

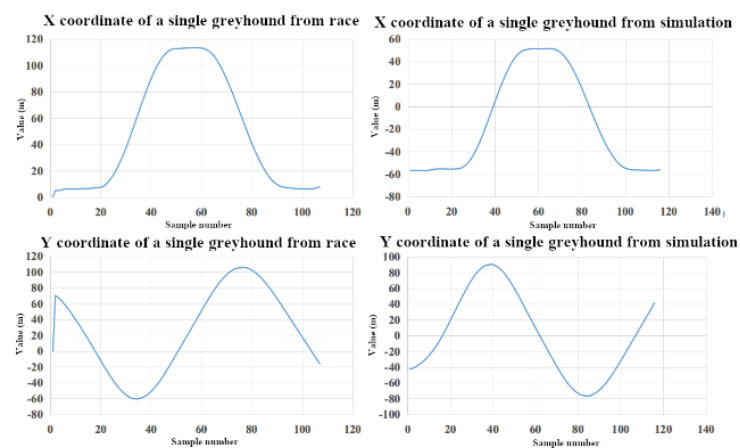

Figure 1: Greyhound coordinate data as produced from simulation and race.

\subsubsection{Speed and Acceleration}

During a race a greyhound's speed remains variable and has different phases as found from simulation and race data. The following graphs depict greyhound's instantaneous speeds versus time for both simulated and actual races. As shown in Figures 2 and 3, the greyhound has an initial high acceleration phase which puts a greyhound into its maximum speed limit of roughly $19.5 \mathrm{~m} / \mathrm{s}$ where the overall duration of this phase depends on race distance, track shape and starting box location in the track. Both simulated and actual race data show initial acceleration continues for $5 \mathrm{~s}$ where the rate change of this initial acceleration is highly variable for actual race whereas for simulation it is fixed as the greyhound model functions use a constant average maximum acceleration for greyhounds. After an initial acceleration phase reaching maximum speed greyhounds tend to lose speed as the time passes as shown in the Figures 2 and 3. The average deceleration of greyhound in this phase is approximately $0.13 \mathrm{~m} / \mathrm{s}^{2}$ as found from both simulation and race. Finally, the local fluctuations in greyhounds speed can be attributed to factors including track surface effects, bumping and checking of greyhounds, and specific individual greyhound behaviour.

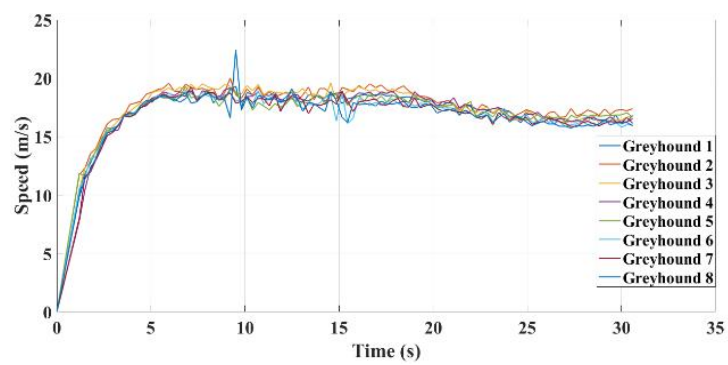

Figure 2: Greyhound speed during a race.

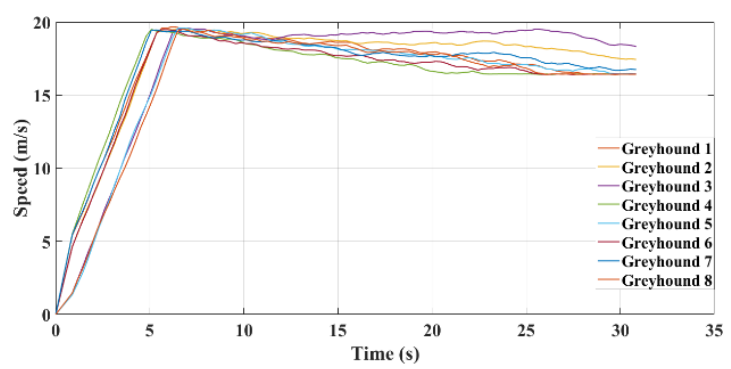

Figure 3: Greyhound speed during a race from simulation.

\subsubsection{Yaw Rate}

Yaw rate is an important aspect in greyhound kinematics as it defines how quickly a greyhound is turning its heading. Also, veering performance during a race as well as the lateral force magnitude acting on a greyhound can be traced from the yaw rates. Figures 4 and 5 illustrate instantaneous yaw rate of a greyhound as derived from simulated and actual races data. For the race distance selected, there are two bends of constant radius in the track which are visible in the yaw rates Figure 4 (red regions) where a yaw rate of approximately $0.37 \mathrm{rad} / \mathrm{s}$ means the greyhound is having a turning of radius roughly $50.5 \mathrm{~m}$ while traversing through the bends with a speed roughly 18.8 $\mathrm{m} / \mathrm{s}$. Also, it is noticeable there is an initial spike in the yaw rate of about $0.4 \mathrm{rad} / \mathrm{s}$ at $2.84 \mathrm{~s}$ and $1.49 \mathrm{~s}$ for simulation and race data respectively. This is because race distance start box location is not perfectly aligned with the track path which causes the greyhound to make an initial sharp turn for transitioning into the track. The local fluctuations in the yaw rates can be caused by greyhound path deviation because of bumping and checking or collision avoiding. Overall, the simulated race shows an excellent agreement with the actual race data. 


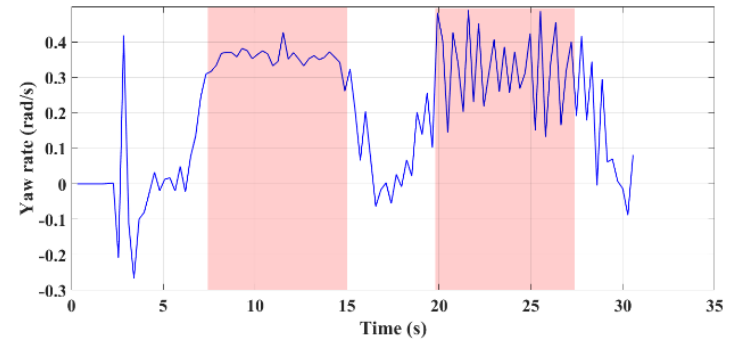

Figure 4: A greyhound's yaw rate during a race from simulation.

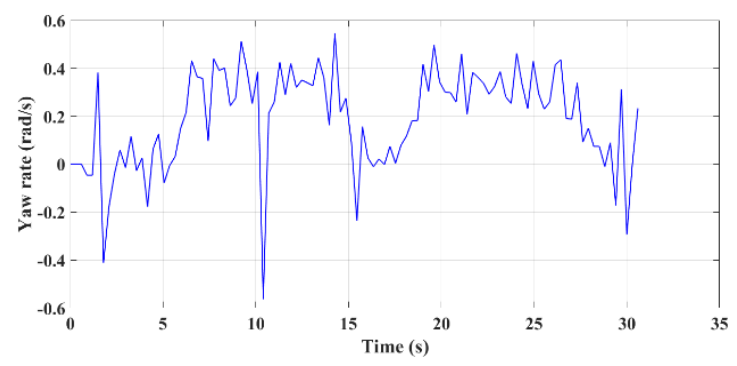

Figure 5: A greyhound's yaw rate during a race.

\subsection{Lure Performance Indicators}

The following major lure performance variables were analysed.

\subsubsection{Speed}

Lure driving condition was analysed between simulation lure model and the actual race. The simulation model yielded better lure driving performance than the actual race as shown in Figures 6 and 7. The overall rate change of lure speed is higher in the actual race and lower in the simulation model. Furthermore, in actual race lure speed was affected by track shape such as around the bends the overall speed was slightly lower whereas no such trends are noticeable in the simulation model other than fluctuations from overall speed.

Finally, it is expected that both simulation model and actual race lure driving would be slightly different from each other as every race is unique in terms of greyhound kinematics which lure highly depended on.

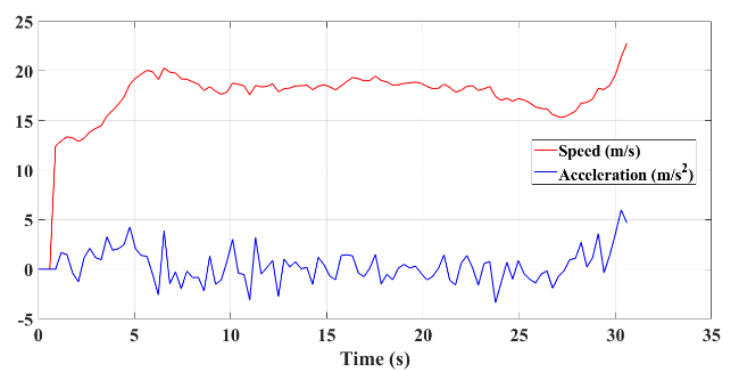

Figure 6: Lure driving speed during a race.

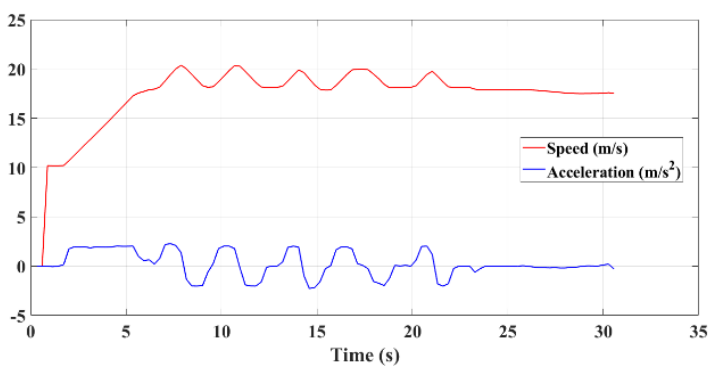

Figure 7: Lure driving speed during a race from simulation.

\subsubsection{Lure Separation Distance}

Maintaining a safe distance from the leading greyhound in the race is also a performance indicator for lure driving condition. The following figures shows the simulation model and actual race lure separation distance from leading greyhound during races. As can be seen, in the actual race lure separation distance tend to become lower and lower until $20 \mathrm{~s}$ into the race where it was increased and lowered again. In the simulation model, lure separation distance is steadier after initial phase and was increased after approximately $21 \mathrm{~s}$ into the race.

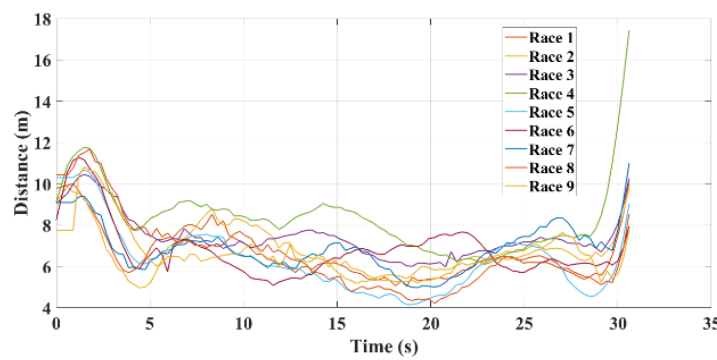

Figure 8: Lure separation distance from different races. 


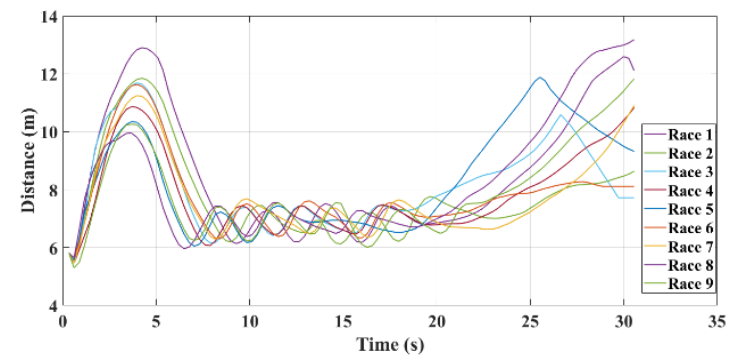

Figure 9: Lure separation distance from different simulated races.

\subsection{Greyhound Racing Performance Indicators}

To measure the racing performance the following major variables were analysed.

\subsubsection{Mean Distance from Cluster Centroid}

In the race, greyhounds pack can be considered as a cluster where the distances of each individual greyhound from the cluster centroid can be averaged to calculate the mean distance from cluster centroid. Mean distance from cluster centroid can be used as a measure to identify greyhounds pack formation and resulting pack density. Figures 10 and 11 show differences between simulated and actual race mean distance from cluster centroid. Evidently, in actual race greyhounds pack remained tightly packed as indicated by the low mean distance from cluster centroid value of about $2 \mathrm{~m}$ until $7 \mathrm{~s}$ into the race and then mean distance saw a gradual increase until the end of the race. In simulation, greyhounds pack density was reduced more rapidly in the first $5 \mathrm{~s}$ into the race and then it saw a slow and gradual increase until the end of the race. Overall, both simulation and actual race showed a similar trend in the greyhound pack density.

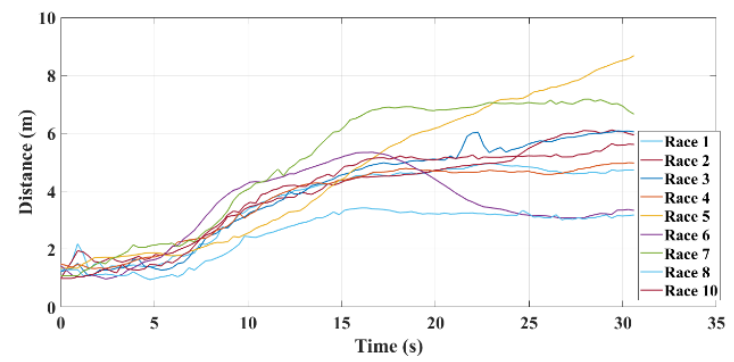

Figure 10: Greyhounds mean distance from cluster centroid for different races.

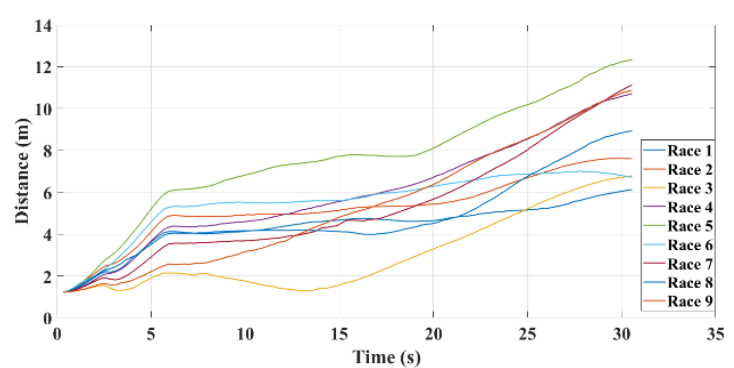

Figure 11: Greyhounds mean distance from cluster centroid for different simulated races.

\subsubsection{Mean Distance from Lure}

Like lure separation distance, the mean distance from lure is the average of all individual greyhound distances from the lure during a race. A higher value of the mean distance from lure indicates greyhounds more spread out along the track while going through different packs formations. As can be seen from the Figure 12, in actual race mean distance from lure slowly increases after initial greyhound acceleration phase. In the simulation, after the initial acceleration phase, the mean distance from lure remains mostly steady until approximately $15 \mathrm{~s}$ into the race while after this period it increases significantly. As a result, both simulation and actual race show that mean distance from lure increases with time during a race which indicates that dispersing of greyhounds is proportional to race time.

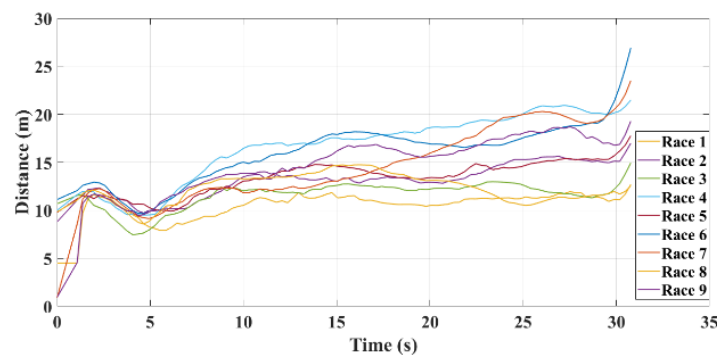

Figure 12: Greyhounds mean distance from lure for different races.

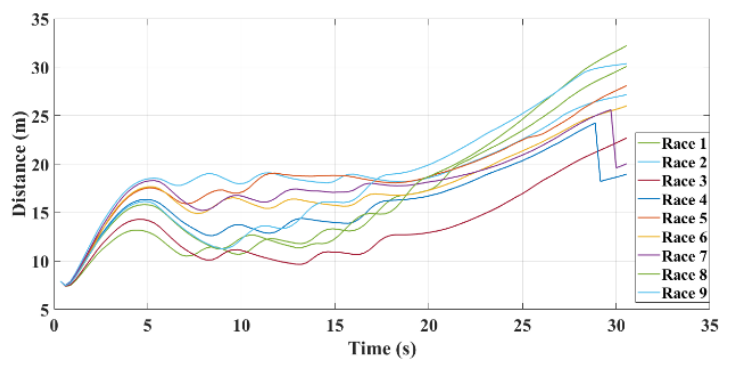

Figure 13: Greyhounds mean distance from lure for different simulated races. 


\subsubsection{Speed vs. Separation Distance}

Both simulation and actual race data showed that there is an approximate inversely proportional relationship between lure speed and lure separation distance which is shown in Figures 14 and 15. The instantaneous increase in lure speed decreases the lure separation distance and vice versa. This observed race dynamic nature can be utilised to design race dynamic nature outcomes.

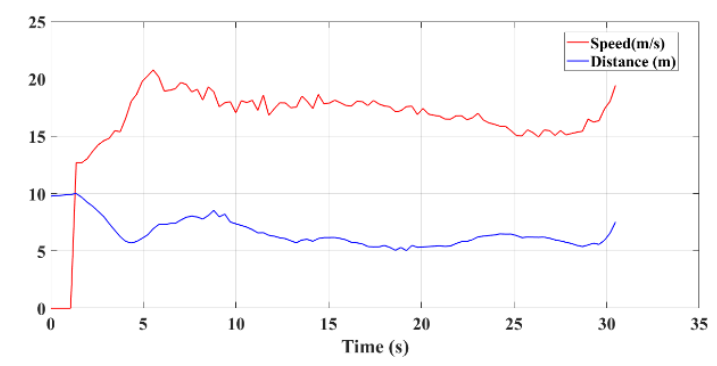

Figure 14: Lure speed vs. lure separation distance during a race.

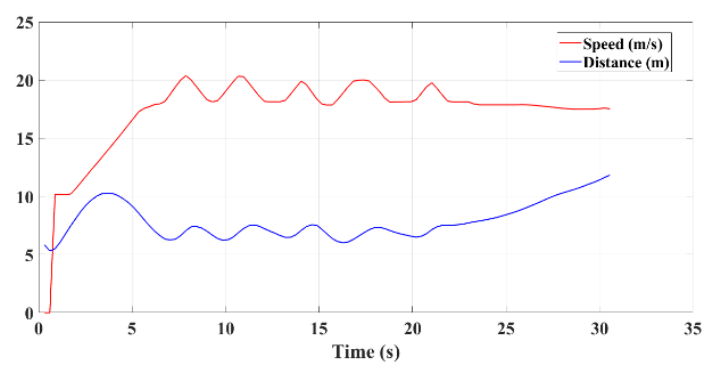

Figure 15: Lure speed vs. lure separation distance during a race from simulation.

\subsubsection{Lure Speed vs. Mean Distance from Cluster Centroid}

Both simulation and real race data indicated no influence of instantaneous lure speed on mean distance from cluster centroid dynamic results.

\subsubsection{Lure Speed vs. Mean Distance from Lure}

Both simulation and real race data did not show any direct relationship between instantaneous lure speed and mean distance from lure variables.

\subsubsection{Lure Separation Distance vs. Mean Separation Distance}

Simulation and actual race data pointed out that there is an approximately proportional relationship between instantaneous lure separation distance and mean distance from lure which is depicted in Figures 16 and 17. Also, the mean distance from lure is always greater than lure separation distance. This relationship suggests that the lure separation distance can be used for managing greyhounds' congestions to some extent as indicated by the mean distance from lure variable.

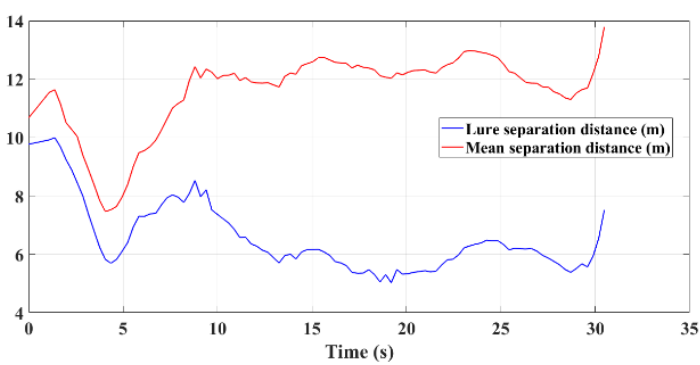

Figure 16: Lure separation vs. mean separation distances during a race.

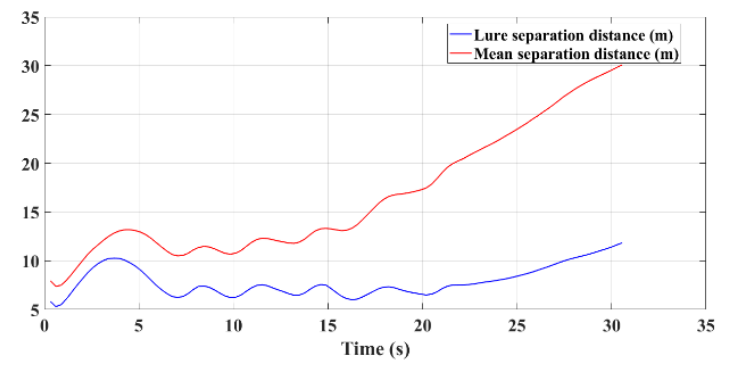

Figure 17: Lure separation vs. mean separation distances during a race from simulation.

\section{CONCLUSIONS}

The greyhound racing simulation models were primarily developed to investigate various factors affecting greyhound racing performance where different racing factors were incorporated as different numerical models to produce racing simulations. By analysing field and racing data the models were refined over time and reached a certain level of maturity where the outputs from the models' simulation showing comparable results to actual race data. The findings from the greyhound racing models simulation suggest trends which are existing in greyhound racing as well as racing factors which would require optimisations. Lastly, this paper presents an approach for developing numerical models for simulation of greyhound racing which can be considered for simulating systems having multiple factors and interacting elements. 


\section{ACKNOWLEDGEMENTS}

This work is sponsored by Greyhound Racing NSW, Australia and Faculty of Engineering and Information Technology at the University of Technology, Sydney, Australia. Special Thanks to Greyhound Racing Victoria, Australia for providing with real time race data and track survey plan.

\section{REFERENCES}

Griebel, G., 2010. Numerical Simulation in Molecular Dynamics: Numeric, Algorithms, Parallelization, Applications, (s.n.).

Garcia, J., 2012. Kinematic and Dynamic Simulation of Multibody Systems: The Real-Time Challenge. SpringerVerlag New York, LLC.

Hayati H., Eager D., Jusufi A., Brown T., A novel approach to analysing rapid tetrapod locomotion using inertia measurement units and stride length as a speed indicator in fast quadrupeds, International Society of Biomechanics Conference, Brisbane, Australia, 23-27 July 2017.

Hayati H., Eager D., Stevenson R., Brown T., Arnott E., The impact of track related parameters on catastrophic injury rate of racing greyhounds, $9^{\text {th }}$ Australian Congress on Applied Mechanics ACAM9, Sydney Australia 27-29 November 2017.

Iddon, J., Lockyer, R. H., and Frean, S. P., 2014, "The effect of season and track condition on injury rate in racing greyhounds," J Small Animal Practise, 55(8), pp. 399404.

Respondek, J.S. 2010, 'Numerical simulation in the partial differential equations: controllability analysis with physically meaningful constraints', Mathematics and Computers in Simulation, 81 (1), pp. 120-132.

Respondek, J.S. 2005, 'Controllability of dynamical systems with constraints', Systems \& Control Letters, 54 (4), pp. 293-314.

Krasny, D.P. and Orin, D.E. 2004, 'Generating high-speed dynamic running gaits in a quadruped robot using an evolutionary search', IEEE Transactions on Systems, Man, and Cybernetics, Part B: Cybernetics, 34, pp. 1685-1696.

Mahadavi F., Hossain I., Hayati H., Eager D., Kennedy P., Track shape, resulting dynamics and injury rates of greyhounds, ASME-IEMCE 2018, Pittsburgh, Pennsylvania, USA, 9-15 November, 2018.

Sicard, G., Short, K. and Manley, P. 1999, 'A survey of injuries at five greyhound racing tracks', Journal of Small Animal Practice, vol. 40, pp. 428-432.

\section{APPENDIX}

\section{Simulation video links}

Race 525 m run 1:

https://drive.google.com/open?id=10nm-

qZGX4ngWrrA3Wn44aU1OOO7V_ALx

Race 525 m run 2:

https://drive.google.com/open?id=1beipV48R5KI6VB8Q

Q2nmlWp6lvNhquoT

Race $525 \mathrm{~m}$ run 3:

https://drive.google.com/open?id=1qHftUiCULA0xnXG-h-hZzL83XZSgxZe

Race $525 \mathrm{~m}$ run 4:

https://drive.google.com/open?id=1ellWXHoksw8M_bSP saJCJv1IowFN1V-W

Race 525 m run 5:

https://drive.google.com/open?id=1FlXH0h2gk5nt33agQy mJAfW2oto48xUK

Race 525 m run 6:

https://drive.google.com/open?id=1_EeYqArAOG2Le1uY yeYHwMGXidoHx_Qe

Race 525 m run 7:

https://drive.google.com/open?id=1gs6EvbaZuCakKSg-

c6YGwixEAWBKXVPB

Race 525 m run 8:

https://drive.google.com/open?id=1ZZ_edPkhlY2_Ayubm jsb7zXp9pTK7fHi

Race 525 m run 9 :

https://drive.google.com/open?id=1zdAQ2KDky5g1ewVI 0yYfahH_2G3-ID5M 